\title{
On Kerov polynomials for Jack characters
}

\author{
Maciej Dołęga非 and Valentin Féray非 \\ ${ }^{1}$ Instytut Matematyczny, Uniwersytet Wroctawski, pl. Grunwaldzki 2/4, 50-384 Wroctaw, Poland \\ ${ }^{2}$ LaBRI, Université Bordeaux 1, 351 cours de la Libération, 33400 Talence, France
}

\begin{abstract}
We consider a deformation of Kerov character polynomials, linked to Jack symmetric functions. It has been introduced recently by M. Lassalle, who formulated several conjectures on these objects, suggesting some underlying combinatorics. We give a partial result in this direction, showing that some quantities are polynomials in the Jack parameter $\alpha$ with prescribed degree.

Our result has several interesting consequences in various directions. Firstly, we give a new proof of the fact that the coefficients of Jack polynomials expanded in the monomial or power-sum basis depend polynomially in $\alpha$. Secondly, we describe asymptotically the shape of random Young diagrams under some deformation of Plancherel measure.

Résumé. On considère une déformation des polynômes de Kerov pour les caractères du groupe symétrique. Cette déformation est liée aux polynômes de Jack. Elle a été récemment définie par M. Lassalle, qui a proposé plusieurs conjectures sur ces objets, suggérant ainsi l'existence d'une combinatoire sous-jacente. Nous donnons un résultat partiel dans cette direction, en montrant que certaines quantités sont des polynômes (dont on contrôle les degrés) en fonction du paramètre de Jack $\alpha$.

Notre résultat a des conséquences intéressantes dans des directions diverses. Premièrement, nous donnons une nouvelle preuve de la polynomialité (toujours en fonction de $\alpha$ ) des coefficients du développement des polynômes de Jack dans la base monomiale. Deuxièmement, nous décrivons asymptotiquement la forme de grands diagrammes de Young distribués selon une déformation de la mesure de Plancherel.
\end{abstract}

Keywords: Jack polynomials; Kerov's polynomials; free cumulants; Young diagrams

\section{Introduction}

\subsection{Polynomiality of Jack polynomials}

In a seminal paper [9], H. Jack introduced a family of symmetric functions $J_{\lambda}^{(\alpha)}$ depending on an additional parameter $\alpha$ called Jack polynomials. Up to multiplicative constants, for $\alpha=1$, Jack polynomials coincide with Schur polynomials. Over the time, it has been shown that several results concerning Schur polynomials can be generalized in a rather natural way to Jack polynomials (Section (VI,10) of I.G. Macdonald's book [20] gives a few results of this kind).

\footnotetext{
${ }^{\dagger}$ This paper is an extended abstract of [4], which will be submitted elsewhere.

${ }^{\ddagger}$ Research of MD is supported by the grant of National Center of Sciences 2011/03/N/ST1/00117 for the years 2012-2014 and by the contract with Uniwersytet Wrocławski 1380/M/IM/11.

$\S$ Partially supported by ANR project PSYCO.

1365-8050 @ 2013 Discrete Mathematics and Theoretical Computer Science (DMTCS), Nancy, France
} 
One of the most surprising features of Jack polynomials is that they have several equivalent classical definitions, but none of them makes obvious the fact that the coefficients of their expansion on the monomial basis are polynomials in $\alpha$ (by construction, they are only rational functions). This property has been established by Lapointe and Vinet [16]. One of the result of this paper is a new proof of Lapointe-Vinet theorem.

Theorem 1.1 (Lapointe and Vinet [16]) The coefficients of the expansion of Jack polynomials in the monomial basis are polynomials in $\alpha$.

This theorem is proved in Section 3.2. We believe that this new proof is interesting in itself, because it relies on a very different approach to Jack polynomials.

To be comprehensive on the subject, let us mention that the coefficients of these polynomials are in fact non-negative integers. This result had been conjectured by R. Stanley and I. Macdonald; see e.g. [20, VI, equation (10.26?)]. It was proved by Knop and Sahi [15], shortly after Lapointe-Vinet's paper. We are unfortunately unable to prove this stronger result with our methods.

\subsection{Dual approach}

We will later define Jack character to be equal (up to some simple normalization constant) to the coefficient $\left[p_{\mu}\right] J_{\lambda}$ in the expansion of the Jack polynomial $J_{\lambda}$ in the basis of power-sum symmetric functions. The idea of the dual approach is to consider Jack characters as a function of $\lambda$ and not as a function of $\mu$ as usual. In more concrete words, we would like to express the Jack character as a sum of some quantities depending on $\lambda$ over some combinatorial set depending on $\mu$ (in Knop-Sahi's result, it is roughly the opposite).

Inspired by the case $\alpha=1$ (which corresponds to the usual characters of the symmetric groups), Lassalle [18] suggested to express Jack characters in terms of, so called, free cumulants of the transition measure of the Young diagram $\lambda$. This expression, called Kerov polynomials for Jack characters, involves rational functions in $\alpha$, which are conjecturally polynomials with non-negative integer coefficients in $\alpha$ and $\beta=1-\alpha$ (see [18, Conjecture 1.2]); we refer to this as Lassalle's conjecture. This suggests the existence of a combinatorial interpretation. A result of this type holds true in the case $\alpha=1$, see [5].

In this paper, we prove a part of Lassalle's conjecture, that is the polynomiality in $\alpha$ (but neither the non-negativity, nor the integrity) of the coefficients.

Theorem 1.2 The coefficients of Kerov polynomials for Jack characters are polynomials in $\alpha$ with rational coefficients.

This theorem with a precise bound on the degree of these polynomials is stated in Section 3.1. In this extended abstract, we only give the guidelines of the proof.

\subsection{Applications}

Our bounds for degrees of coefficients of Kerov polynomials for Jack characters imply in particular that some coefficients (corresponding to the leading term for some gradation) are independent on $\alpha$. In Section 4. we use this simple remark to describe asymptotically the shape of random Young diagrams whose distribution is a deformation of Plancherel measure.

Another consequence of our results is a uniform proof of the polynomiality of structure constants of several meaningful algebras. This allows us to solve some conjectures of Matsumoto [21] and to give a partial answer to the Matching-Jack conjecture of I. Goulden and D. Jackson [7]. Due to the lack of space, we will not present these results in this extended abstract. They can be found in [4, Section 4]. 
Outline of the paper. Section 2 gives all necessary definitions and background; in particular we recall the notions of free cumulants and Kerov polynomials. In Section 3 we sketch the proof of Theorem 1.2 and Theorem 1.1. Finally, we state and prove our results on large Young diagrams in Section 4

\section{Jack characters and Kerov polynomials}

\subsection{Partitions and symmetric functions}

We begin with a few classical definitions and notations.

A partition $\lambda$ of $n$ (denote it by $\lambda \vdash n$ ) is a non-increasing list $\left(\lambda_{1}, \ldots, \lambda_{\ell}\right)$ of positive integers of sum equal to $n$. Then $n$ is called the size of $\lambda$ and denoted by $|\lambda|$ and the number $\ell$ is the length of the partition (denoted by $\ell(\lambda)$ ).

We also consider the graded ring of symmetric functions Sym. Recall that its homogeneous component $\operatorname{Sym}_{n}$ of degree $n$ admits several classical bases: the monomials $\left(m_{\lambda}\right)_{\lambda \vdash n}$, the power-sums $\left(p_{\lambda}\right)_{\lambda \vdash n}$, each indexed by partitions of $n$. All the definitions can be found in [20, Chapter I].

Jack polynomials are symmetric functions indexed by partitions and depending on a parameter $(\alpha)$. There exist several normalizations for Jack polynomials in the literature. We shall work with the one denoted by $J$ in the book of Macdonald [20, VI, (10.22)] and use the same notation as he does. For a fixed value of the parameter $\alpha$, the family $\left(J_{\lambda}^{(\alpha)}\right)_{\lambda \vdash n}$ forms a basis of $\operatorname{Sym}_{n}$.

\subsection{Jack characters}

As power-sum symmetric functions $\left(p_{\rho}\right)_{\rho \vdash n}$ form a basis of $\mathrm{Sym}_{n}$, we can expand the Jack polynomial $J_{\lambda}^{(\alpha)}$ in that base. For $\lambda \vdash n$, there exist (unique) coefficients $\theta_{\rho}^{(\alpha)}(\lambda)$ such that

$$
J_{\lambda}^{(\alpha)}=\sum_{\substack{\rho: \\|\rho|=|\lambda|}} \theta_{\rho}^{(\alpha)}(\lambda) p_{\rho} .
$$

Then we can define Jack characters by the formula:

$$
C h_{\mu}^{(\alpha)}(\lambda)=\alpha^{-\frac{|\mu|-\ell(\mu)}{2}}\left(\begin{array}{c}
|\lambda|-|\mu|+m_{1}(\mu) \\
m_{1}(\mu)
\end{array}\right) z_{\mu} \theta_{\mu,|>|-|\mu|}^{(\alpha)}(\lambda),
$$

where $m_{i}(\mu)$ denotes the multiplicity of $i$ in the partition $\mu$ and $z_{\mu}=\mu_{1} \mu_{2} \cdots m_{1}(\mu) ! m_{2}(\mu) ! \cdots$.

In the case $\alpha=1$, Jack polynomials correspond, up to some normalization constants, to Schur symmetric functions. The coefficients of the latter in the basis of the power-sum symmetric functions are known to be equal to the irreducible characters of the symmetric groups; see [20, Section I,7] (this explains the name characters in the general case, even if, except for $\alpha=1 / 2,1,2$, these quantities have no known representation-theoretical interpretation). It means that Jack characters with parameter $\alpha=1$ correspond, up to some numerical factors, to character values of the symmetric groups.

This normalization corresponds in fact to the one used by Kerov and Olshanski in [13]. These normalized characters - following the denomination of Kerov and Olshanski - have plenty of interesting properties; for example when considered as functions on the set of Young diagrams $\lambda \mapsto C h_{\mu}^{(1)}(\lambda)$, they form a linear basis (when $\mu$ runs over the set of all partitions) of the algebra $\Lambda^{\star}$ of shifted symmetric functions, which is very rich in structure.

Jack characters have been first considered by M. Lassalle in [17]. Note that the normalization used here is different that the one of these papers. The reason of this new choice of normalization will be clear later. 


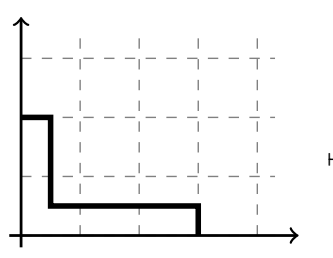

$\lambda$

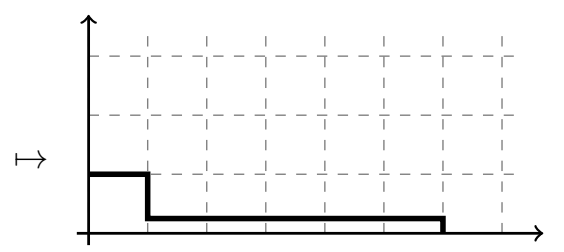

$T_{2, \frac{1}{2}}(\lambda)$

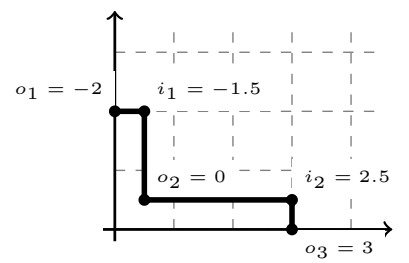

Fig. 2: A generalized Young diagram $L$ with

Fig. 1: Example of stretched Young diagram.

the corresponding set $\mathbb{O}_{L}$ and $\mathbb{I}_{L}$.

\subsection{Generalized Young diagrams and Kerov interlacing coordinates}

In this section, we will see different ways of representing Young diagrams and even more general objects related to them. Let us consider a zigzag line $L$ going from a point $(0, y)$ on the $y$-axis to a point $(x, 0)$ on the $x$-axis. We assume that every piece is either an horizontal segment from left to right or a vertical segment from top to bottom. A Young diagram can be seen as such a zigzag line: just consider its border. Therefore, we call these zigzag lines generalized Young diagrams.

We will be in particular interested in the following generalized Young diagrams. Let $\lambda$ be a (generalized) Young diagram and $s$ and $t$ two positive real numbers. We denote by $T_{s, t}(\lambda)$ the broken line obtained by stretching $\lambda$ horizontally by a factor $s$ and vertically by a factor $t$ (see Figure 1 , we use french convention to draw Young diagrams). These anisotropic Young diagrams have been introduced by S. Kerov in [11]. In the special case $s=t$, we denote by $D_{s}(\lambda)=T_{s, s}(\lambda)$ the dilated Young diagram.

The content of a point of a plane is the difference of its $x$-coordinate and its $y$-coordinate. We denote by $\mathbb{O}_{L}$ the sets of contents of the outer corners of $L$, that is corners which are points of $L$ connecting a horizontal line on the left with vertical line on the bottom. Similarly, the set $\mathbb{I}_{L}$ is defined as the contents of the inner corners, that is corners which are points of $L$ connecting a horizontal line on the right with vertical line above. An example is given on Figure 2. The denomination inner/outer may seem strange, but it refers to the fact that the box in the corner is inside or outside the diagram.

A generalized Young diagram can also be seen as a function on the real line. Indeed, if one rotates the zigzag line counterclockwise by $45^{\circ}$ and scale it by a factor $\sqrt{2}$ (so that the new $x$-coordinate corresponds to contents), then it can be seen as the graph of a piecewise affine continuous function with slope \pm 1 . We denote this function by $\omega(\lambda)$. Therefore, we shall call continuous Young diagram a Lipshitz continuous function $\omega$ with Lipshitz constant 1 such that $\omega(x)=|x|$ for $|x|$ big enough . This notion will be used in Section 4 to describe the limit shape of Young diagrams.

\subsection{Polynomial functions on the set of Young diagrams}

If $k$ is a positive integer, one can consider the power sum symmetric function $p_{k}$, evaluated on the difference of alphabets $\mathbb{O}_{L}-\mathbb{I}_{L}$. By definition, it is a function on generalized Young diagrams given by:

$$
L \mapsto p_{k}\left(\mathbb{O}_{L}-\mathbb{I}_{L}\right):=\sum_{o \in \mathbb{O}_{L}} o^{k}-\sum_{i \in \mathbb{I}_{L}} i^{k} .
$$

As any symmetric function can be written (uniquely) in terms of $p_{k}$, we can define $f\left(\mathbb{O}_{L}-\mathbb{I}_{L}\right)$ for any symmetric function $f$ as follows. Expand $f$ on the power-sum basis $f=\sum_{\rho} a_{\rho} p_{\rho_{1}} \cdots p_{\rho_{\ell}}$ for some 
family of scalars $\left(a_{\rho}\right)$ indexed by partitions. Then, by definition

$$
f\left(\mathbb{O}_{L}-\mathbb{I}_{L}\right)=\sum_{\rho \text { partition }} a_{\rho} p_{\rho_{1}}\left(\mathbb{O}_{L}-\mathbb{I}_{L}\right) \cdots p_{\rho_{\ell}}\left(\mathbb{O}_{L}-\mathbb{I}_{L}\right) .
$$

This convenient notation is classical in lambda-ring calculus.

Consider the set of functions $\left\{\lambda \mapsto f\left(\mathbb{O}_{L}-\mathbb{I}_{L}\right)\right\}$, where $f$ describes the set of symmetric functions. This is a subalgebra of the algebra of functions on the set of all Young diagrams. Following S. Kerov and G. Olshanski, we shall call it the algebra of polynomial functions and denote it by $\Lambda^{\star}$.

V. Ivanov and G. Olshanski [8, Corollary 2.8] have shown that the normalized characters $\left(\lambda \mapsto C h_{\mu}^{(1)}(\lambda)\right)_{\mu}$ form a linear basis of this algebra and $\left(\lambda \mapsto p_{k}\left(\mathbb{O}_{\lambda}-\mathbb{I}_{\lambda}\right)\right)_{k>2}$ forms an algebraic basis of $\Lambda^{\star}$ (for all diagrams $\lambda$, one has $p_{1}\left(\mathbb{O}_{\lambda}-\mathbb{I}_{\lambda}\right)=0$ ). This algebra admits several other characterization: for instance it corresponds to the algebra of shifted symmetric functions (see [8, Section 1 and 2]).

All this has a natural extension for a general parameter $\alpha$.

We say that $F$ is an $\alpha$-polynomial function on the set of (generalized) Young diagrams if

$$
\lambda \mapsto F\left(T_{\sqrt{\alpha}}^{-1}, \sqrt{\alpha}(\lambda)\right)
$$

is a polynomial function. The ring of $\alpha$ polynomial functions is denoted by $\Lambda_{\star}^{(\alpha)}$. Then $\left(\lambda \mapsto C h_{\mu}^{(\alpha)}(\lambda)\right)_{\mu}$ forms a linear basis of $\Lambda_{\star}^{(\alpha)}$. This is a consequence of a result of M. Lassalle [17, Proposition 2].

Remark 1 Lassalle's result is in fact formulated in terms of shifted symmetric functions, but as mentioned above, it is proved in [8] Section 1 and 2] that they correspond to polynomial functions.

Fact 2 With the definitions above, it should be clear that polynomial functions are defined on generalized Young diagrams. They can in fact also be canonically extended to continuous Young diagrams; see [1. Section 1.2]. This will be useful in Section 4.

\subsection{Transition measure and free cumulants}

$\mathrm{S}$. Kerov [10] introduced the notion of transition measure of a Young diagram. This probability measure $\mu_{\lambda}$ associated to $\lambda$ is defined by its Cauchy transform

$$
G_{\mu_{\lambda}}(z)=\int_{\mathbb{R}} \frac{d \mu_{\lambda}(x)}{z-x}=\frac{\prod_{i \in \mathbb{I}_{\lambda}} z-i}{\prod_{o \in \mathbb{O}_{\lambda}} z-o} .
$$

Its moments are $h_{k}\left(\mathbb{O}_{\lambda}-\mathbb{I}_{\lambda}\right)$, where $h_{k}$ is the complete symmetric function of degree $k$, hence they are polynomial functions on the set of Young diagrams; we will denote them by $M_{k}^{(1)}$.

In Voiculescu's free probability it is very convenient to associate to a probability measure $\mu$ a sequence of numbers $\left(R_{k}(\mu)\right)_{k \geq 1}$ called free cumulants [26]. The free cumulants of the transition measure of Young diagrams appeared first in the work of P. Biane [1] and play an important role in the asymptotic representation theory. As explained by M. Lassalle (look at the case $\alpha=1$ of [18, Section 5]), they can be expressed as

$$
R_{k}^{(1)}(\lambda):=R_{k}\left(\mu_{\lambda}\right)=e_{k}^{\star}\left(\mathbb{O}_{\lambda}-\mathbb{I}_{\lambda}\right)
$$

for some homogeneous symmetric function $e_{k}^{\star}$ of degree $k$. Note also that $\left(e_{k}^{\star}\right)_{k}$ as well as complete symmetric functions $\left(h_{k}\right)_{k}$ are algebraic basis of symmetric functions and, hence $\left(R_{k}^{(1)}\right)_{k \geq 2}$ as well as 
$\left(M_{k}^{(1)}\right)_{k \geq 2}$ are algebraic basis of ring of polynomial functions on the set of Young diagrams $\left(R_{1}^{(1)}=M_{1}^{(1)}\right.$ is the null function).

Fact 3 It is easy to see that, as $h_{k}$ and $e_{k}^{\star}$ are homogeneous symmetric functions, the corresponding polynomial functions $M_{k}^{(1)}$ and $R_{k}^{(1)}$ are compatible with dilations. Namely

$$
M_{k}^{(1)}\left(D_{s}(\lambda)\right)=s^{k} M_{k}^{(1)}(\lambda) ; \quad R_{k}^{(1)}\left(D_{s}(\lambda)\right)=s^{k} R_{k}^{(1)}(\lambda) .
$$

Using the relevant definitions, the $\alpha$-anisotropic moments and free cumulants defined by

$$
\begin{gathered}
M_{k}^{(\alpha)}(\lambda)=M_{k}^{(1)}\left(T_{\sqrt{\alpha}, \sqrt{\alpha}^{-1}}(\lambda)\right), \\
R_{k}^{(\alpha)}(\lambda)=R_{k}^{(1)}\left(T_{\sqrt{\alpha}, \sqrt{\alpha}^{-1}}(\lambda)\right)
\end{gathered}
$$

are $\alpha$-polynomial and the families $\left(M_{k}^{(\alpha)}\right)_{k \geq 2}$ and $\left(R_{k}^{(\alpha)}\right)_{k \geq 2}$ are two algebraic basis of the algebra $\Lambda_{(\alpha)}^{\star}$.

\subsection{Kerov polynomials}

Recall that Jack characters $C h_{\mu}^{(\alpha)}$ are $\alpha$-polynomial functions hence can be expressed in terms of the two algebraic bases above.

Definition-Proposition 2.1 Let $\mu$ be a partition and $\alpha>0$ be a fixed real number. There exist unique polynomials $L_{\mu}^{(\alpha)}$ and $K_{\mu}^{(\alpha)}$ such that, for every $\lambda$,

$$
\begin{aligned}
& C h_{\mu}^{(\alpha)}(\lambda)=L_{\mu}^{(\alpha)}\left(M_{2}^{(\alpha)}(\lambda), M_{3}^{(\alpha)}(\lambda), \cdots\right), \\
& C h_{\mu}^{(\alpha)}(\lambda)=K_{\mu}^{(\alpha)}\left(R_{2}^{(\alpha)}(\lambda), R_{3}^{(\alpha)}(\lambda), \cdots\right) .
\end{aligned}
$$

The polynomials $K_{\mu}^{(\alpha)}$ have been introduced by S. Kerov in the case $\alpha=1$ [12] and by M. Lassalle in the general case [18]. Once again, we emphasize that our normalizations are different from his.

From now on, when it does not create any confusion, we suppress the superscript $(\alpha)$.

We present a few examples of polynomials $K_{\mu}$. This data has been computed using the one given in [18, page 2230]

$$
\begin{aligned}
K_{(1)} & =R_{2}, \\
K_{(2)} & =R_{3}+\gamma R_{2}, \\
K_{(3)} & =R_{4}+3 \gamma R_{3}+\left(1+2 \gamma^{2}\right) R_{2}, \\
K_{(4)} & =R_{5}+\gamma\left(6 R_{4}+R_{2}^{2}\right)+\left(5+11 \gamma^{2}\right) R_{3}+\left(7 \gamma+6 \gamma^{3}\right) R_{2}, \\
K_{(2,2)} & =R_{3}^{2}+2 \gamma R_{3} R_{2}-4 R_{4}+\left(\gamma^{2}-2\right) R_{2}^{2}-10 \gamma R_{3}-\left(6 \gamma^{2}+2\right) R_{2} .
\end{aligned}
$$

where we set $\gamma=\frac{1-\alpha}{\sqrt{\alpha}}$. A few striking facts appear on these examples:

- All coefficients are polynomials in the auxiliary parameter $\gamma$ : the sketch of the proof of this fact will be presented in the next section with explicit bounds on the degrees. 
- For one part partition, polynomials $K_{(r)}$ have non-negative coefficients. We are unfortunately unable to prove this statement, which is a more precise version of [18, Conjecture 1.2]. A similar conjecture holds for several part partitions, see [18, Conjecture 1.2].

Remark 4 This facts explain our changes of normalization. In Lassalle's work, the non-negativity of the coefficients is hidden: he has to use two variables $\alpha$ and $\beta=1-\alpha$ and choose a "natural" way to write all quantities in terms of $\alpha$ and $\beta$. Using our normalizations and the parameter $\gamma$, the non-negativity of the coefficients appears directly.

\section{Polynomiality}

\subsection{Main result}

Theorem 3.1 The coefficient of $M_{\rho}$ in Jack character polynomial $L_{\mu}$ is a polynomial in $\gamma$ of degree smaller or equal to

$$
\min (|\mu|+\ell(\mu)-|\rho|,|\mu|-\ell(\mu)-(|\rho|-2 \ell(\rho))) .
$$

Moreover, it has the same parity as the integer $|\mu|+\ell(\mu)-|\rho|$.

The same is true for the coefficient of $R_{\rho}$ in $K_{\mu}$.

We do not prove this theorem in this extended abstract. The proof is of course available in the long version of the paper [4, Section 3]. Here, we are going to present a guidelines of this proof.

The difficulty is that the proof of the existence of the polynomials $L_{\mu}$ and $K_{\mu}$ (Proposition 2.1p is not constructive. However, M. Lassalle gives an algorithm to compute the polynomial $K_{\mu}$ [18, Section 9], but his algorithm involves an induction on the size of the partition $|\mu|$. The coefficients of $K_{\mu}$ are the solutions of an overdetermined linear system involving the coefficients of $K_{\mu^{\prime}}$, for some partitions $\mu^{\prime}$ with $\left|\mu^{\prime}\right|<|\mu|$. His algorithm can be easily adapted to $L_{\mu}$ [4, Section 3.2].

Our proof relies on this work and on the two following important facts:

- the linear system computing the coefficients of $L_{\mu}$ contains a triangular subsystem (this is not true with $K_{\mu}$ );

- with our normalization of Jack characters and anisotropic moments, the diagonal coefficients of this linear subsystem are independent of $\gamma$ (and hence invertible in $\mathbb{Q}[\gamma]$ ).

The polynomiality in $\gamma$ follows from these two facts. To obtain the bound on the degree, one has to look carefully at the degrees of the coefficients of the linear system.

Recall that our normalization is different from the one used by M. Lassalle. After a simple rewriting game [4, Section 3.6], we can see that Theorem 3.1 implies that the coefficients of $L_{\mu}$ and $K_{\mu}$ with Lassalle's normalizations are polynomials in $\alpha$ (that is the statement of Theorem 1.2.

\subsection{Lapointe-Vinet theorem}

In this section, we prove that $\theta_{\mu}(\lambda)$ is a polynomial in $\alpha$. This result was already known (see Introduction), but in our opinion it illustrates that Lassalle's approach to Jack polynomials is relevant.

To deduce this from the results above, one has to see how $M_{k}(\lambda)$ depends on $\alpha$.

Lemma 3.2 Let $k \geq 2$ be an integer and $\lambda$ be a partition. Then $\sqrt{\alpha}^{k-2} M_{k}(\lambda)$ is a polynomial in $\alpha$ with integer coefficients. 
Proof: We use induction over $|\lambda|$. Let $o=(x, y)$ be an outer corner of $\lambda$, we denote by $\lambda^{(o)}$ the diagram obtained from $\lambda$ by adding a box at place $o$. Comparing the corner of $\lambda^{(o)}$ and $\lambda$, we get that:

$$
\mathbb{O}_{\lambda^{(o)}}-\mathbb{I}_{\lambda^{(o)}}=\mathbb{O}_{\lambda}-\mathbb{I}_{\lambda}+\{x-(y+1)\}+\{x+1-y\}-\{x-y\}
$$

(for readers not used to $\lambda$-ring, this equality can be understood as equality between formal sums of elements in the set). After dilatation, we get

$\mathbb{O}_{T_{\sqrt{\alpha}, \sqrt{\alpha}-1}\left(\lambda^{(o)}\right)}-\mathbb{I}_{T_{\sqrt{\alpha}, \sqrt{\alpha}-1}\left(\lambda^{(o)}\right)}=\mathbb{O}_{T_{\sqrt{\alpha}, \sqrt{\alpha}-1}(\lambda)}-\mathbb{I}_{T_{\sqrt{\alpha}, \sqrt{\alpha}-1}(\lambda)}+\left\{z_{o}-\frac{1}{\sqrt{\alpha}}\right\}+\left\{z_{o}+\alpha\right\}-\left\{z_{o}\right\}$,

and $z_{o}=\sqrt{\alpha} x-y / \sqrt{\alpha}$ is the content of the considered corner in $T_{\sqrt{\alpha}, \sqrt{\alpha}}(\lambda)$.

By a standard $\lambda$-ring computations (see [18, Proposition 8.3]), this yields

$$
M_{k}\left(\lambda^{(o)}\right)-M_{k}(\lambda)=\sum_{\substack{r \geq 1, s, t \geq 0, 2 r+s+t \leq k}} z_{o}^{k-2 r-s-t}\left(\begin{array}{c}
k-t-1 \\
2 r+s-1
\end{array}\right)\left(\begin{array}{c}
r+s-1 \\
s
\end{array}\right)(-\gamma)^{s} M_{t}(\lambda)
$$

which can be rewritten as

$$
\begin{aligned}
\sqrt{\alpha}^{k-2} M_{k}\left(\lambda^{(o)}\right)-\sqrt{\alpha}^{k-2} M_{k}(\lambda)= & \sum_{\substack{r \geq 1, s, t \geq 0, 2 r+s+t \leq k}} \alpha^{r}\left(\sqrt{\alpha} z_{o}\right)^{k-2 r-s-t} \\
& \left(\begin{array}{c}
k-t-1 \\
2 r+s-1
\end{array}\right)\left(\begin{array}{c}
r+s-1 \\
s
\end{array}\right)(\alpha-1)^{s} \sqrt{\alpha}^{t-2} M_{t}(\lambda) .
\end{aligned}
$$

But $\sqrt{\alpha} z_{o}=\alpha x-y$ is a polynomial in $\alpha$ with integer coefficients. Thus the induction is immediate.

Now we write, for $\mu, \lambda \vdash n$,

$$
z_{\mu} \theta_{\mu}(\lambda)=\alpha^{\frac{|\mu|-\ell(\mu)}{2}} C h_{\mu}(\lambda)=\sum_{\rho} \alpha^{\frac{|\mu|-\ell(\mu)-(|\rho|-2 \ell(\rho))}{2}} a_{\rho}^{\mu}\left(\prod_{i \leq \ell(\rho)} \sqrt{\alpha}^{\rho_{i}-2} M_{\rho_{i}}(\lambda)\right) .
$$

The quantities $\alpha^{\frac{|\mu|-\ell(\mu)-(|\rho|-2 \ell(\rho))}{2}} a_{\rho}^{\mu}$ and $\sqrt{\alpha}^{\rho_{i}-2} M_{\rho_{i}}(\lambda)$ are polynomials in $\alpha$ (by Theorem 3.1 and Lemma 3.2, hence $\theta_{\mu}(\lambda)$ is a polynomial in $\alpha$, which proves Theorem 1.1

\subsection{Gradation}

Looking at Theorem 3.1 it makes natural to consider some gradations on $\Lambda_{\star}^{(\alpha)}$. This structure will also be useful in the next section.

The ring $\Lambda_{\star}^{(\alpha)}$ of $\alpha$-polynomial functions can be endowed with a gradation by deciding that $M_{k}$ is a homogeneous function of degree $k$ : as $\left(M_{k}\right)_{k \geq 2}$ is an algebraic basis of $\Lambda_{\star}^{(\alpha)}$, any choice of degree for $M_{k}$ (for all $k \geq 2$ ) defines uniquely a gradation on $\Lambda_{\star}^{(\alpha)}$. Then $R_{k}$ is also a homogeneous function of degree $k$, thanks to the moment-free cumulant relations, see e.g. [1, Section 2.4].

Theorem 3.1 shows that $C h_{\mu}$ has at most degree $|\mu|+\ell(\mu)$ (this has also been proved by M. Lassalle [18, Proposition 9.2 (ii)]). Note that $C h_{\mu}$ is not homogeneous in general. Moreover, its component of 
degree $|\mu|+\ell(\mu)$ does not depend on $\alpha$. As this dominant term is known in the case $\alpha=1$ (see for example [24, Theorem 4.9]), one obtains the following result (which extends [18, Theorem 10.2]):

$$
C h_{\mu}=\prod_{i=1}^{\ell(\mu)} R_{\mu_{i}+1}+\text { smaller degree terms. }
$$

In particular, $C h_{\mu}$ has exactly degree $|\mu|+\ell(\mu)$.

Consider the subspace $V_{\leq d} \subset \Lambda_{\star}^{(\alpha)}$ of elements of degree less or equal to $d$. Its dimension is the number of partitions $\rho$ of size less or equal to $d$ with no parts equal to 1. By removing 1 from every part of $\rho$, we see that this is also the number of partitions $\mu$ such that $|\mu|+\ell(\mu) \leq d$. But the latter index the functions $C h_{\mu}$ lying in $V_{\leq d}$. Hence,

$$
V_{\leq d}=\operatorname{Vect}\left(\left\{C h_{\mu},|\mu|+\ell(\mu) \leq d\right\}\right)
$$

and the degree of an element in $\Lambda_{\star}^{(\alpha)}$ can be determined as follows:

$$
\operatorname{deg}\left(\sum_{\mu} a_{\mu} C h_{\mu}\right)=\max _{\mu: a_{\mu} \neq 0}|\mu|+\ell(\mu) .
$$

Remark 5 The algebra $\Lambda_{\star}^{(\alpha)}$ admits other relevant gradations, see [4] Sections 3.5 and 3.8].

\section{Application: asymptotics of large Young diagrams}

We consider the following deformation of the Plancherel measure $\mathbb{P}_{n}^{(\alpha)}(\lambda)=\frac{\alpha^{n} n !}{j_{\lambda}^{(\alpha)}}$, where $j_{\lambda}^{(\alpha)}$ is the following deformation of the square of the hook products:

$$
j_{\lambda}^{(\alpha)}=\prod_{\square \in \lambda}(\alpha a(\square)+\ell(\square)+1)(\alpha a(\square)+\ell(\square)+\alpha) .
$$

Here, $a(\square)$ and $\ell(\square)$ are respectively the arm and leg length of the box as defined in [20, Chapter I]. The probability measure $\mathbb{P}_{n}^{(\alpha)}$ on Young diagrams of size $n$ has appeared recently in several research papers [3, 6, 23, 21] and is presented as an important area of research in Okounkov's survey on random partitions [22, §3.3]. When $\alpha=1$, it specializes to the well-known Plancherel measure for the symmetric groups.

The following property, which corresponds to the case $\pi=\left(1^{n}\right)$ in [21, Equation (8.4)], characterizes the Jack-Plancherel measure:

$$
\mathbb{E}_{\mathbb{P}_{n}^{(\alpha)}}\left(\theta_{\mu}^{(\alpha)}(\lambda)\right)=\delta_{\mu, 1^{n}}
$$

where $\lambda$ is a random variable distributed according to $\mathbb{P}_{n}^{(\alpha)}$.

Using the definition of $C h_{\mu}$ we have:

$$
\mathbb{E}_{\mathbb{P}_{n}^{(\alpha)}}\left(C h_{\mu}\right)= \begin{cases}n(n-1) \cdots(n-k+1) & \text { if } \mu=1^{k} \text { for some } k \leq n, \\ 0 & \text { otherwise }\end{cases}
$$

As $C h_{\mu}$ is a linear basis of $\Lambda_{\star}^{(\alpha)}$, it implies the following lemma (which is an analogue of [23, Theorem 5.5] with another gradation). 
Lemma 4.1 Let $F$ be an $\alpha$-polynomial function. Then $\mathbb{E}_{\mathbb{P}_{n}^{(\alpha)}}(F)$ is a polynomial in $n$ of degree at most $\operatorname{deg}(F) / 2$.

Proof: It is enough to verify this lemma on the basis $C h_{\mu}$ because of equation (2). But in this case $\mathbb{E}_{\mathbb{P}_{n}^{(\alpha)}}(F)$ is explicit (see formula above) and the lemma is obvious (recall that $\operatorname{deg}\left(C h_{\mu}\right)=|\mu|+\ell(\mu)$; see Section 3.3.

Let $\left(\lambda^{n}\right)_{n \geq 1}$ be a sequence of random partitions, where $\lambda^{n}$ has distribution $\mathbb{P}_{n}^{(\alpha)}$. In the case $\alpha=1$, it has been proved in 1977 separately by Logan and Shepp [19] and Kerov and Vershik [14] that, in probability,

$$
\lim _{n \rightarrow \infty}\left\|\omega\left(D_{1 / \sqrt{n}}\left(\lambda^{n}\right)\right)-\Omega\right\|=0
$$

where $\Omega$ is the limit shape given explicitly as follows:

$$
\Omega(x)= \begin{cases}|x| & \text { if }|x| \geq 2 ; \\ \frac{2}{\pi}\left(x \cdot \arcsin \frac{x}{2}+\sqrt{4-x^{2}}\right) & \text { otherwise. }\end{cases}
$$

Recall from Section 2.3 that $D_{s}(\lambda)$ is the Young diagram $\lambda$ dilated by a factor $s$ and $\omega(\lambda)$ is by definition the function whose graphical representation is the border of $\lambda$, rotated by $45^{\circ}$ (see Section 2.3 ) and stretched by $\sqrt{2}$.

In the general $\alpha$ case, we have the following weak convergence result:

Proposition 4.2 For any 1-polynomial function $F \in \Lambda_{\star}^{(1)}$, when $n$ tends to infinity, one has

$$
F\left(T_{\sqrt{\alpha / n}, 1 / \sqrt{n \alpha}}\left(\lambda^{n}\right)\right) \stackrel{\mathbb{P}_{n}^{(\alpha)}}{\longrightarrow} F(\Omega),
$$

where $\stackrel{\mathbb{P}_{n}^{(\alpha)}}{\longrightarrow}$ means convergence in probability.

Proof: As $\left(R_{k}^{(1)}\right)_{k \geq 2}$ is an algebraic basis of $\Lambda_{\star}^{(1)}$, it is enough to prove the proposition for any $R_{k}^{(1)}$.

Let $\mu$ be partition. As mentioned at the beginning of the section, one has:

$$
\prod_{i \leq \ell(\mu)} R_{\mu_{i}+1}^{(\alpha)}=C h_{\mu}+\text { terms of degree at most }|\mu|+\ell(\mu)-1 .
$$

Together with Lemma 4.1 and the formula for $\mathbb{E}_{\mathbb{P}_{n}^{(\alpha)}}\left(C h_{\mu}\right)$, this implies:

$$
\mathbb{E}_{\mathbb{P}_{n}^{(\alpha)}}\left(\prod_{i \leq \ell(\mu)} R_{\mu_{i}+1}^{(\alpha)}\right)= \begin{cases}n(n-1) \cdots(n-k+1)+O\left(n^{k-1}\right) & \text { if } \mu=1^{k} \text { for some } k \\ o\left(n^{\frac{|| \mid+\ell(\mu)}{2}}\right) & \text { otherwise }\end{cases}
$$

In particular, one has that

$$
\begin{aligned}
\mathbb{E}_{\mathbb{P}_{n}^{(\alpha)}}\left(R_{k}^{(\alpha)}\left(D_{1 / \sqrt{n}}\left(\lambda^{n}\right)\right)\right) & =\frac{1}{n^{k / 2}} \mathbb{E}_{\mathbb{P}_{n}^{(\alpha)}}\left(R_{k}^{(\alpha)}\right)=\delta_{k, 2}+O\left(\frac{1}{\sqrt{n}}\right) \\
\operatorname{Var}_{\mathbb{P}_{n}^{(\alpha)}}\left(R_{k}^{(\alpha)}\left(D_{1 / \sqrt{n}}\left(\lambda^{n}\right)\right)\right) & =\frac{1}{n^{k}}\left(\mathbb{E}_{\mathbb{P}_{n}^{(\alpha)}}\left(\left(R_{k}^{(\alpha)}\right)^{2}\right)-\mathbb{E}_{\mathbb{P}_{n}^{(\alpha)}}\left(R_{k}^{(\alpha)}\right)^{2}\right)=O\left(\frac{1}{n}\right) .
\end{aligned}
$$


Thus, for each $k, R_{k}^{(\alpha)}\left(D_{1 / \sqrt{n}}\left(\lambda^{n}\right)\right)$ converges in probability towards $\delta_{k, 2}$. But, by definition

$$
R_{k}^{(\alpha)}\left(D_{1 / \sqrt{n}}\left(\lambda^{n}\right)\right)=R_{k}^{(1)}\left(T_{\sqrt{\alpha / n}, 1 / \sqrt{n \alpha}}\left(\lambda^{n}\right)\right)
$$

and $\left(\delta_{k, 2}\right)_{k \geq 2}$ is the sequence of free cumulants of the continuous diagram $\Omega$ (see [2, Section 3.1]), i.e. $\delta_{k, 2}=R_{k}^{(1)}(\Omega)$.

Roughly speaking, Proposition 4.2 means that, the stretched Young diagram $T_{\sqrt{\alpha / n}, 1 / \sqrt{n \alpha}}\left(\lambda^{n}\right)$ converges weakly towards $\Omega$ (in probability). So this result already means that the considered diagrams admit some limit shape.

However, it would be desirable to obtain a result with uniform convergence, which is a more natural notion of convergence. This can be done thanks to the following lemma.

Lemma 4.3 There exists a constant $C$ such that

$$
\lim _{n \rightarrow \infty} \mathbb{P}\left[\max \left(\frac{c\left(\lambda^{n}\right)}{\sqrt{n}} ; \frac{r\left(\lambda^{n}\right)}{\sqrt{n}}\right) \leq C\right]=1,
$$

where, for each $n$, the diagram $\lambda^{n}$ is chosen randomly with distribution $\mathbb{P}_{n}^{(\alpha)}$ and $r\left(\lambda^{n}\right)$ and $c\left(\lambda^{n}\right)$ are respectively its numbers of rows and columns.

The proof of this lemma is quite technical and relies on the explicit formula 3 for $\mathbb{P}_{n}^{(\alpha)}$. It can be found in [4, Section 6.4]. We can now state the uniform convergence result.

Theorem 4.4 For each $n$, let $\lambda^{n}$ be a random Young diagram of size $n$ distributed with $\alpha$-Plancherel measure. Then, in probability,

$$
\lim _{n \rightarrow \infty}\left\|\omega\left(T_{\sqrt{\alpha / n}, 1 / \sqrt{n \alpha}}\left(\lambda^{n}\right)\right)-\Omega\right\|=0 .
$$

Proof: It follows from Proposition 4.2 and Lemma 4.3 by the same argument as the one given in $[8$, Theorem 5.5].

The idea of using polynomial functions to study the asymptotic shape of Young diagrams has been developped by S. Kerov (see [8]). In the case $\alpha=1$, he gave more precise result that what we have here: he proved that for any polynomial function $F$, the quantity $F\left(\lambda^{n}\right)$ has Gaussian fluctuations. A better understanding of polynomials $K_{\mu}$ could lead to a proof of a similar phenomena in the general $\alpha$ case, using the ideas introduced in [25]. Let us mention the existence of a partial result (corresponding to $F=C h^{(2)}$ ) obtained by J. Fulman [6, Theorem 1.2] by another method.

\section{References}

[1] P. Biane. Representations of symmetric groups and free probability. Adv. Math., 138(1):126-181, 1998.

[2] P. Biane. Approximate factorization and concentration for characters of symmetric groups. Internat. Math. Res. Notices, 2001(4):179-192, 2001.

[3] A. Borodin and G. Olshanski. Z-measures on partitions and their scaling limits. European Journal of Combinatorics, 26(6):795-834, 2005. 
[4] M. Dołęga and V. Féray. On Kerov polynomials for Jack characters. arXiv preprint 1201.1806, 2012.

[5] M. Dołęga, V. Féray, and P. Śniady. Explicit combinatorial interpretation of Kerov character polynomials as numbers of permutation factorizations. Adv. Math., 225(1):81-120, 2010.

[6] J. Fulman. Stein's method, Jack measure, and the Metropolis algorithm. Journal of Combinatorial Theory, Series A, 108(2):275-296, 2004.

[7] I. P. Goulden and D. M. Jackson. Connection coefficients, matchings, maps and combinatorial conjectures for Jack symmetric functions. Trans. Amer. Math. Soc., 348(3):873-892, 1996.

[8] V. Ivanov and G. Olshanski. Kerov's central limit theorem for the Plancherel measure on Young diagrams. In Symmetric functions 2001: surveys of developments and perspectives, volume 74 of NATO Sci. Ser. II Math. Phys. Chem., pages 93-151. Kluwer Acad. Publ., Dordrecht, 2002.

[9] H. Jack. A class of symmetric polynomials with a parameter. Proc. Roy. Soc. Edinburgh Sect. A, 69:1-18, 1970/1971.

[10] S. Kerov. Transition probabilities of continual Young diagrams and the Markov moment problem. Funct. Anal. Appl., 27(3):104-117, 1993.

[11] S. Kerov. Anisotropic Young diagrams and Jack symmetric functions. Funct. Anal. Appl., 34:45-51, 2000.

[12] S. Kerov. Talk in Institute Henri Poincaré. Paris, January 2000.

[13] S. Kerov and G. Olshanski. Polynomial functions on the set of Young diagrams. Comptes rendus de l'Académie des sciences. Série 1, Mathématique, 319(2):121-126, 1994.

[14] S. Kerov and A. Vershik. Asymptotics of the Plancherel measure of the symmetric group and the limiting form of Young tables. Soviet Math. Dokl, 18:527-531, 1977.

[15] F. Knop and S. Sahi. A recursion and a combinatorial formula for Jack polynomials. Inventiones Mathematicae, 128(1):9-22, 1997

[16] L. Lapointe and L. Vinet. A Rodrigues formula for the Jack polynomials and the Macdonald-Stanley conjecture. International Mathematics Research Notices, 1995(9):419-424, 1995.

[17] M. Lassalle. A positivity conjecture for Jack polynomials. Math. Res. Lett., 15(4):661-681, 2008.

[18] M. Lassalle. Jack polynomials and free cumulants. Adv. Math., 222(6):2227-2269, 2009.

[19] B. F. Logan and L. A. Shepp. A variational problem for random Young tableaux. Advances in Math., 26(2):206222, 1977.

[20] I. G. Macdonald. Symmetric functions and Hall polynomials. Oxford Mathematical Monographs. The Clarendon Press Oxford University Press, New York, second edition, 1995. With contributions by A. Zelevinsky, Oxford Science Publications.

[21] S. Matsumoto. Jucys-Murphy elements, orthogonal matrix integrals, and Jack measures. The Ramanujan Journal, 26(1):69-107, 2010.

[22] A. Okounkov. The uses of random partitions. In Fourteenth International Congress on Mathematical Physics, pages 379-403. Word Scientists, 2003.

[23] G. Olshanski. Plancherel averages: Remarks on a paper by Stanley. Elec. Jour. Comb., 17(1):R43, 2010.

[24] P. Śniady. Asymptotics of characters of symmetric groups, genus expansion and free probability. Discrete Math., 306(7):624-665, 2006.

[25] P. Śniady. Gaussian fluctuations of characters of symmetric groups and of Young diagrams. Probab. Theory Related Fields, 136(2):263-297, 2006.

[26] D. Voiculescu. Addition of certain noncommuting random variables. J. Funct. Anal., 66(3):323-346, 1986. 